\title{
Design of Load Shedding Schemes against Voltage Instability
}

\author{
C. Moors \\ Research engineer, FRIA \\ University of Liège, Institut Montefiore \\ Sart Tilman B28, B-4000 Liège, Belgium
}

\author{
D. Lefebvre \\ Hydro-Québec, Division Transénergie \\ CP 10000 Montreal (QC), Canada
}

\author{
T. Van Cutsem \\ Research director, FNRS \\ University of Liege, Institut Montefiore \\ Sart Tilman B28, B-4000 Liège, Belgium
}

\begin{abstract}
This paper proposes a methodology for the design of automatic load shedding against long-term voltage instability. In a first step, a set of training scenarios is set up, corresponding to various operating conditions and disturbances. Each scenario is analyzed to determine the minimal load shedding which stabilizes the system, with due consideration for the shedding location and delay. In a second step, the parameters of a closed-loop undervoltage load shedding scheme are determined so as to: (i) approach as closely as possible the optimal sheddings computed in the first step, over the whole set of scenarios; (ii) stabilize the system in all the unstable scenarios and (iii) shed no load in the stable ones. The corresponding optimization problem is solved using a (micro-)Genetic Algorithm. A detailed example is given on the Hydro-Québec system in which load shedding is presently planned.
\end{abstract}

Keywords. System stability and analysis, voltage stabilization and control, genetic algorithms.

\section{INTRODUCTION}

There are two lines of defence against incidents which jeopardize the stability of power systems:

- preventively: analyze the system security margins with respect to credible contingencies, i.e. incidents with a reasonable probability of occurrence, and take appropriate preventive actions to restore sufficient margins when needed;

- correctively: implement automatic corrective actions, through System Protection Schemes (SPS) ${ }^{1}$ to face the more severe, but less likely incidents.

The preventive security criteria usually require that the system remains stable after any credible contingency, without the help of corrective actions. The main reason is that these actions usually affect the system generation and/or load, which is acceptable only in the presence of severe disturbances.

The present paper concentrates on long-term voltage stability, driven by Load Tap Changers (LTCs), generator OverExcitation Limiters (OELs), switched shunt compensation, restorative loads, and possibly secondary voltage control. This type of instability has become a major threat in many systems $[1,2]$.

\footnotetext{
'also referred to as Special Protection Schemes
}

Since long-term voltage instability is triggered mainly by the loss of generation or transmission facilities, " $\mathrm{N}-1$ " contingencies corresponding to the loss of a single equipment are usually considered in preventive security analysis. On the other hand, $\mathrm{N}-2$ and more severe disturbances should be counteracted by an SPS. While it must be used in the last resort and to the least extent, automatic load shedding is very effective in this respect.

A few undervoltage load shedding schemes have been implemented throughout the world (e.g. [3]. Beside time-domain numerical simulation, methods have been proposed to identify the best location, time and amount of shedding in a given unstable scenario (e.g $[4,5])$. There is however a need for a methodology to help planners in designing this type of SPS.

This paper proposes such a methodology. The latter consists of two steps:

- in the first step, a set of training scenarios is set up, corresponding to various operating conditions and various disturbances. Each scenario is analyzed to determine the minimal load shedding which stabilizes the system, with due consideration for the shedding delay;

- in the second step, the parameters of a closed-loop protection are determined in order to approach as closely as possible the optimal sheddings computed in the first step, over the whole set of scenarios. A genetic algorithm is used to this purpose.

The paper is organized as follows. Section II describes how the minimal load shedding is determined when analyzing the unstable scenarios in the first step. Section III deals with the second step of the procedure. Section IV provides a rather complex example taken from the Hydro-Québec system, in which an undervoltage load shedding scheme is planned. The paper ends up with some concluding remarks.

\section{DETERMINATION OF OPTIMAL LOAD SHEDDING}

Location, amount and delay are the three main characteristics of load shedding. Obviously the amount of load shedding should be minimal.

For a given shedding delay and location, the minimal amount of shedding $P^{\min }$ can be simply determined by binary or incremental search, resorting to time-domain simulations to check the system behaviour. As far as long-term voltage stability is concerned, the computing times can be dramatically reduced by using the Quasi Steady-State (QSS) simulation techique. This well-documented approach is based on time decomposition and consists of replacing the short-term dynamics by equilibrium equations, while focusing on the long-term dynamics $[2,6]$. 
The next point is to determine which delay and location yield the smallest amount $P^{m i n}$. These two problems are discussed separately hereafter.

\section{A. Optimizing with respect to the shedding delay}

The main motivation for delaying load shedding is to ascertain that the system is indeed voltage unstable, and hence to avoid shedding load unduly.

The influence of the delay $\tau$ (counted from the disturbance inception) on the minimal amount of power to shed $P^{\min }$ can be easily established for the simple two-bus system of Fig. 1.a. In this system, the load is assumed to obey the well-known exponential model in the short term and to restore to constant power in the long term, owing to the LTC effect. The $P^{\min }$ vs. $\tau$ characteristic is shown with solid line in Fig. 1.b and is easily explained as follows [5]:

- the minimal amount of load power to shed does not vary as far as load shedding takes place before the critical time $\tau_{c}$. This amount, denoted $P^{*}$, is the load decrease just needed to create a long-term equilibrium in the post-contingency configuration; - if the shedding takes place after $\tau_{c}$, more load has to be shed; this is a matter of attraction towards the newly created long-term equilibrium.

Note however that a severe disturbance may yield the characteristic shown with dashed line in Fig. 1.b, in which $P^{\text {min }}$ increases right away after the disturbance.

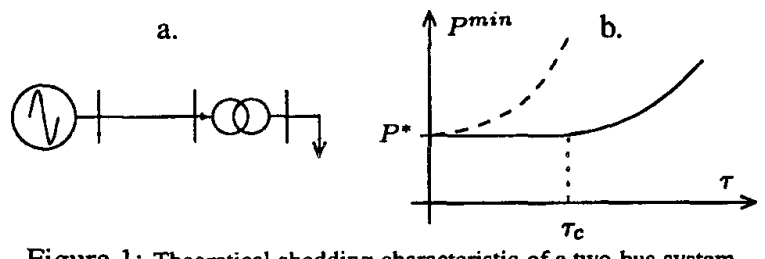

Figure 1: Theoretical shedding characteristic of a two-bus system

According to the authors' experience, large real-life systems have $P^{\min }$ vs. $\tau$ characteristics quite close to that of Fig. 1, as far as the long-term dynamics are governed by OELs, LTCs and secondary voltage control (if any) [5].

On the other hand, the characteristic may change when other post-contingency controls "compete" with load shedding. In this case, it may be advantageous to delay load shedding so that these controls act first and hence less load is shed. An example is provided in Fig. 2, relative to the Hydro-Québec system considered in Section IV. In this system, automatic shunt reactor tripping significantly contributes to stabilizing the system in its post-contingency configuration. The figure shows that $280 \mathrm{MW}$ load are saved when the shedding is delayed by 16 seconds, allowing 2970 Mvar to be tripped before load is shed.

In the design of a load shedding protection, we will use the minimum $P^{*}$ as a target value. When the $P^{\min }$ vs. $\tau$ characteristic is of the type shown in Fig. $1, P^{\star}$ is merely taken as the value of $P^{\text {min }}$ for a load shedding taking place very shortly after the disturbance (ideally $\tau=0$ ). When a more complex characteristic is expected, such as in Fig. 2, $P^{\star}$ is determined as

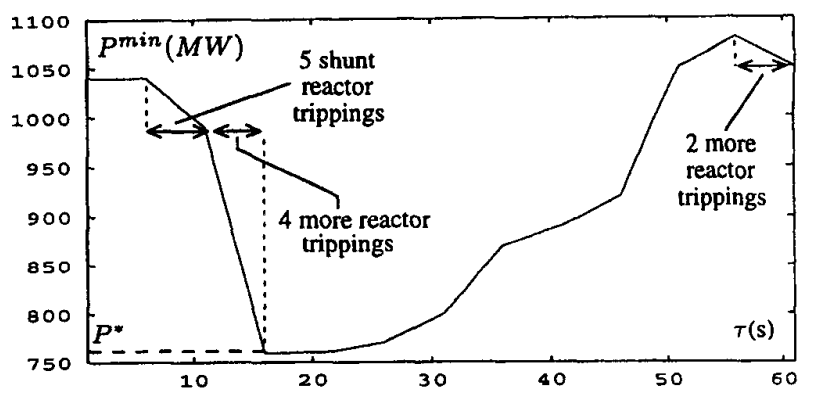

Figure 2: A shedding characteristic of the Hydro-Québec system

the minimum value of $P^{m i n}$ over a speficied interval of $\tau$.

B. Optimizing with respect to the shedding location.

There are basically two proven approaches to identify the optimal location:

- small-disturbance analysis coupled with time-domain simulation [5]. Along the unstable trajectory provided by a timedomain method, sensitivity analysis is used to identify the critical point, at which the eigenvector corresponding to the (almost) zero eigenvalue is computed. This information allows to obtain a ranking of load buses with respect to the efficiency of load shedding. A given amount of load shedding is distributed over the buses in this order, taking into account the interruptible fraction of each load;

- Optimal Power Flow (OPF) [4]. The objective is to minimize the amount of load shedding, taking into account the constraints stemming from the load flow equations, the generator reactive limits and the interruptible fraction of each load. This approach provides the optimal location and the minimal amount of load shedding in a single step.

In the first approach, a coupling between time-domain simulation and small-disturbance analysis is necessary. This coupling in easier with QSS simulation which is free from shortterm transients.

In the OPF approach, it is possible to require the system to satisfy some operating constraints, in addition to being stable. On the other hand, the OPF relies on a simplified system modelling (typically load flow equations) and does not allow to check the delay aspects discussed in the previous section. To this purpose, a time-domain method is needed anyway.

\section{DESIGN OF THE LOAD SHEDDING PROTECTION}

\section{A. Scenario analysis}

As already mentioned, the first step of our approach consists in setting up a set of $s$ training scenarios, corresponding to various topologies, load levels, generation schemes, and contingencies.

In principle all the scenarios to be dealt with by a single protection should involve the same weak area of the system; in other words, the instability modes and hence the optimal 
shedding locations should be rather close for all the unstable scenarios of the set. Therefore, we assume that a common bus ranking can be set up for all of them. Once this ranking has been identified, the minimal amount of load shedding $P_{i}^{\star}$ is determined for each scenario $(i=1, \ldots, s)$, according to the procedure described in the previous section.

\section{B. Logic of the load shedding protection}

Generally speaking SPSs can be classified into :

- event-based vs. response-based protections. The former rely on the direct identification of the disturbance (e.g. circuit breaker operation signals, etc.), while the latter rely on the effect of the disturbance on measured system variables. Event-based protections are needed when speed of action is essential. This is usually not the case for long-term voltage instability; hence, a response-based protection will be considered in the sequel. We assume that a single signal $V$ is monitored. This is typically the average voltage over several transmission buses in the load area of concern. In practice, other measurements can enter the logic, such as the reactive reserve on neighbouring generators, etc. but this is not considered here for simplicity.

- open-loop vs. closed-loop protections. By closed-loop we mean a protection which takes successive actions, each on the basis of the signal $V$ resulting from the previous actions. In other words, the signal $V$ stemming from the system is fed back to the protection. This closed-loop design is preferred as being more robust with respect to modelling and operating condition uncertainties.

In accordance with the above remarks, we consider a protection based on $k$ rules of the type:

if $V$ is smaller than $V_{i}^{\text {min }}$ during $d_{i}$ seconds, shed $\Delta P_{i} M W$ The number $k$ of rules is decided a priori; in practice it is typically equal to 2 or 3 .

Note that such a protection operates in closed loop since $V$ is continuously measured and the same rule may trigger several successive load sheddings in time.

\section{Statement of the design problem}

Given the $s$ training scenarios, the problem is to determine the $3 k$-dimensional vector of unknowns:

$$
\mathbf{x}=\left[V_{1}^{m i n}, d_{1}, \Delta P_{1}, \ldots, V_{k}^{\min }, d_{k}, \Delta P_{k}\right]
$$

such that the following requirements are met:

1. the amount of load shedding must be as close as possible to the minimal amount $P_{i}^{\star} \quad(i=1, \ldots, s)$ determined in the first step;

2. all unstable scenarios must be saved (SPS dependability);

3. no load must be shed in a stable scenario (SPS security);

4. optionally, some other constraints can be imposed. For instance, the distribution voltages should not stay below some threshold for more than some time.

This can be translated into an optimization problem: minimize the discrepancies $P_{i}^{s h}(\mathbf{x})-P_{i}^{\star}$, where $P_{i}^{s h}(\mathbf{x})$ is the total load power shed in the $i$-th scenario, for a given protection set- ting $\mathbf{x}$. Two objectives have been considered:

$$
\begin{aligned}
& \min _{\mathbf{x}} \sum_{i}\left[P_{i}^{s h}(\mathbf{x})-P_{i}^{\star}+p_{i}(\mathbf{x})\right] \\
& \text { or } \min _{\mathbf{x}} \max _{i}\left[P_{i}^{s h}(\mathrm{x})-P_{i}^{\star}+p_{i}(\mathrm{x})\right]
\end{aligned}
$$

where the sum and the max extend over the unstable scenarios and $p_{i}(\mathbf{x})$ is a penalty term accounting for the violation of the above requirements, as described hereafter.

When the system is unstable (requirement 2 violated), transmission voltages eventually become smaller than some threshold $V^{\text {low }}$. Denoting by $t_{\text {low }}$ the time at which this occurs ${ }^{2}$, the penalty takes on the form:

$$
p_{i}=\frac{C_{1}}{t_{l o w}+C_{2}} \quad C_{1} \gg 0 \quad C_{2}>0
$$

When an amount $P_{i}^{s h}$ is shed in a stable case (requirement 3 violated), the penalty term takes on the form:

$$
p_{i}=k P_{i}^{s h} \quad k \gg 1
$$

Let $t_{\text {rec }}$ be the recovery time, i.e. the time at which voltages are again larger than a specified value $V_{\min }$. Requirement 4 consists in specifying that $t_{\text {rec }}$ is smaller than a given value $t_{\text {rec }}^{\max }$. If this does not hold, the penalty is taken as:

$$
p_{i}=C_{3}\left(t_{r e c}-t_{r e c}^{\max }\right) \quad C_{3} \gg 0
$$

Note that with the above penalties, the more dangerous a situation (i.e. the shorter $t_{\text {low }}$, the larger $P_{i}^{s h}$ or $t_{\text {rec }}$ ), the higher the penalty. This is expected to provide the optimization method with information on how to improve the parameters.

The optimization problem (2-6) is complex. Indeed, both $P_{i}^{s h}$ and $p_{i}$ must be determined from time-domain simulations and hence, explicit analytical expressions cannot be established. Moreover, they vary with $\mathbf{x}$ in a discontinuous manner. This prevents from using classical analytical optimization methods. Also, multiple local minima are expected. A "controlled random search" method, in the form of a Genetic Algorithm, seems better suited to this combinatorial optimization problem.

\section{Optimization through a micro-Genetic Algorithm}

Genetic algorithms (GAs) are optimization techniques inspired by the theory of evolution [8]. They combine survival of the fittest among string structures with a structured yet randomized information exchange to form a search algorithm with some of the innovative flair of human search. They allow to find near-optimum solutions of multimodal objective functions.

The basic principle of GA methods is to make evolve a population of potential solutions to a given optimization problem. Specifically, they operate on structures, which are encoded representations of the solution (e.g. strings of bits from a binary alphabet). Each solution is associated with a fitness value, which

\footnotetext{
${ }^{2}$ In a long-term voltage unstable situation, the system may finally loose its short-term equilibrium, which corresponds to a fast collapse When the QSS technique is used, the simulation cannot proceed. If this occurs before the $V^{\text {low }}$ threshold is reached, the last time instant reached is substituted for $t_{\text {low }}$
} 
is simply the corresponding value of the objective function to be optimized. When each structure in the population has been evaluated, a new population of candidate solutions is formed in two steps. First, structures in the current population are selected for replication based on their relative fitness. The higher the fitness value of an individual, the higher its representation in the subsequent generation. Next, the selected structures are altered using crossover and mutation operators. The crossover operator combines the features of two parent structures to form two similar children by exchanging parts of the parents' strings. The mutation operator, which alters randomly one or more components of a selected structure, provides the way to introduce new genetic materials. Mutation usually ensures the reachability of all points in the search space, preventing premature convergence. The resulting children are then evaluated and inserted back into the population, replacing older members.

Progressively, this process improves the performances of the population through the generations, until no better individual can be found. At that stage, the algorithm has converged, and most of the individuals in the population are almost identical. While randomized, genetic algorithms are no simple random walk. They efficiently exploit historical information to speculate on new search points with expected improved performance.

In this work, we have used a micro-Genetic Algorithm ( $\mu \mathrm{GA})$ $[9,10]$. Compared to GAs, $\mu$ GAs have a much smaller population size (typically 5 individuals vs. 30 to 200 individuals for GAs). Moreover, the mutation operator is replaced by a random insertion of new individuals, once convergence of the micro population has been detected. Studies have shown that $\mu \mathrm{GAs}$ reach the near-optimal region much earlier.

\section{E. On the choice of training scenarios}

Attention must be paid to the design of the training scenarios. This task requires a good engineering knowledge of the system. Although the choice of scenarios depends to a large extent on system specifics, the following appear as important guidelines: - in order to meet requirement 3 of Section III.C, the training set should include a significant number of marginally stable cases, on which the protection must be trained not to act;

- the $P_{i}^{\star}$ values should be rather uniformly distributed in between the marginally and the most severely unstable cases;

- with the protection already trained not to act in marginally stable cases, more stable scenarios need not be considered.

\section{RESULTS ON THE HYDRO-QUEBEC SYSTEM}

\section{A. Voltage stability of the Hydro-Québec system}

The Hydro-Québec system is characterized by great distances (more than $1000 \mathrm{~km}$ ) between the large hydro generation areas (James Bay, Churchill Falls and Manic-Outardes) and the main load center (around Montréal and Québec City). Accordingly, the company has developed an extensive $735-\mathrm{kV}$ transmission system, whose lines are located along two main axes. This system is angle stability limited in the North, voltage stability limited in the South (near the load center). Frequency stability is also a concern due to the system interconnection through DC links only, as well as the sensitivity of loads to voltage.

In the recent years, Hydro-Québec has undertaken a major program to upgrade the reliability of its transmission system. In particular a defence plan is being deployed against extreme contingencies [7]. This includes generation rejection and remote load shedding, automatic shunt reactor switching, underfrequency load shedding and in a near future, undervoltage load shedding.

Beside static var compensators and synchronous condensers, the automatic shunt reactor switching devices, known under the French acronym MAIS, play an important rôle in voltage control [11]. These devices, in operation since early 1997, are now available in $22735-\mathrm{kV}$ substations and control a large part of the total 25,500 Mvar shunt compensation. Each MAIS relies on the local voltage, the coordination between substations being performed through the switching delays. While fast-acting MAIS can improve transient (angle) stability, slower MAIS significantly contribute to voltage stability.

Preventive security assessment is also a major concern. Presently, secure operation limits are determined in operational planning, with the help of ASTRE, the QSS simulation program developed at the University of Liège $[2,6]$. This fast tool has been used in the present study. The corresponding model includes around 550 buses, 100 generators and 230 LTCs. The total load is around $33,000 \mathrm{MW}$.

\section{B. Training scenarios and main protection parameters}

The study reported in this paper involves 8 system configurations, summarized in Table 1 .

Table 1. System configurations considered in the training scenarios
\begin{tabular}{|c|c|c|c|}
\hline $\begin{array}{c}\text { confi- } \\
\text { guration }\end{array}$ & $\begin{array}{c}\text { number of } \\
\text { 735-kV lines } \\
\text { out of service }\end{array}$ & $\begin{array}{c}\text { synchronous } \\
\text { condensers }\end{array}$ & $\begin{array}{c}\text { MAIS } \\
\text { devices }\end{array}$ \\
\hline \hline A & 0 & 6 & 5 \\
B & 1 & 6 & 9 \\
C & 1 & 6 & 8 \\
D & 1 & 8 & 16 \\
E & 1 & 8 & 14 \\
F & 4 & 8 & 16 \\
G & 3 & 8 & 14 \\
H & 3 & 8 & 14 \\
\hline
\end{tabular}

Table 2 details the 36 scenarios finally selected. They involve $\mathrm{N}-1, \mathrm{~N}-2$ and $\mathrm{N}-3$ contingencies, respectively. In accordance with the standard operating rules, the system is stable following any N-1 incident. The MAIS devices can be used to this purpose.

In each unstable scenario, the best load shedding location has been identified. Therefrom, a common ranking of load buses has been set up. For simplicity, each load is assumed fully interruptible. Using this bus ranking, the minimal amount of load shedding $P_{i}^{\star}$ required to stabilize the system has been determined in the 19 unstable scenarios. The values, computed with an accuracy of $10 \mathrm{MW}$, are given in Table 2 . The most severe incident requires to shed load at 8 buses. 
Table 2. Description of the 36 training scenarios

\begin{tabular}{|c|c|r|r||r|r|r|r|}
\hline No & conf. & $\begin{array}{r}\text { incid. } \\
\text { type }\end{array}$ & $\begin{array}{r}P_{i}^{\star} \\
(\mathrm{MW})\end{array}$ & No & conf. & $\begin{array}{r}\text { incid. } \\
\text { type }\end{array}$ & $\begin{array}{r}P_{i}^{\boldsymbol{K}} \\
(\mathrm{MW})\end{array}$ \\
\hline 1 & $\mathrm{~A}$ & $\mathrm{~N}-1$ & 0 & 19 & $\mathrm{E}$ & $\mathrm{N}-2$ & 890 \\
2 & $\mathrm{~A}$ & $\mathrm{~N}-1$ & 0 & 20 & $\mathrm{E}$ & $\mathrm{N}-2$ & 890 \\
3 & $\mathrm{~A}$ & $\mathrm{~N}-2$ & 1090 & 21 & $\mathrm{~F}$ & $\mathrm{~N}-1$ & 0 \\
4 & $\mathrm{~A}$ & $\mathrm{~N}-2$ & 460 & 22 & $\mathrm{~F}$ & $\mathrm{~N}-2$ & 0 \\
5 & $\mathrm{~A}$ & $\mathrm{~N}-2$ & 110 & 23 & $\mathrm{~F}$ & $\mathrm{~N}-2$ & 1110 \\
6 & $\mathrm{~A}$ & $\mathrm{~N}-3$ & 1520 & 24 & $\mathrm{~F}$ & $\mathrm{~N}-2$ & 860 \\
7 & $\mathrm{~B}$ & $\mathrm{~N}-1$ & 0 & 25 & $\mathrm{~F}$ & $\mathrm{~N}-2$ & 620 \\
8 & $\mathrm{~B}$ & $\mathrm{~N}-2$ & 200 & 26 & $\mathrm{G}$ & $\mathrm{N}-1$ & 0 \\
9 & $\mathrm{C}$ & $\mathrm{N}-1$ & 0 & 27 & $\mathrm{G}$ & $\mathrm{N}-1$ & 0 \\
10 & $\mathrm{D}$ & $\mathrm{N}-1$ & 0 & 28 & $\mathrm{G}$ & $\mathrm{N}-2$ & 40 \\
11 & $\mathrm{D}$ & $\mathrm{N}-1$ & 0 & 29 & $\mathrm{G}$ & $\mathrm{N}-2$ & 1790 \\
12 & $\mathrm{D}$ & $\mathrm{N}-2$ & 0 & 30 & $\mathrm{G}$ & $\mathrm{N}-2$ & 880 \\
13 & $\mathrm{D}$ & $\mathrm{N}-2$ & 740 & 31 & $\mathrm{G}$ & $\mathrm{N}-2$ & 760 \\
14 & $\mathrm{D}$ & $\mathrm{N}-2$ & 350 & 32 & $\mathrm{H}$ & $\mathrm{N}-1$ & 0 \\
15 & $\mathrm{E}$ & $\mathrm{N}-1$ & 0 & 33 & $\mathrm{H}$ & $\mathrm{N}-1$ & 0 \\
16 & $\mathrm{E}$ & $\mathrm{N}-1$ & 0 & 34 & $\mathrm{H}$ & $\mathrm{N}-2$ & 310 \\
17 & $\mathrm{E}$ & $\mathrm{N}-2$ & 0 & 35 & $\mathrm{H}$ & $\mathrm{N}-2$ & 730 \\
18 & $\mathrm{E}$ & $\mathrm{N}-2$ & 0 & 36 & $\mathrm{H}$ & $\mathrm{N}-2$ & 600 \\
\hline
\end{tabular}

The scenarios have been chosen according to the guidelines of Section III.E: the 17 scenarios with $P_{i}^{\star}=0$ are marginally stable situations, while the nonzero values of $P_{i}^{*}$ range rather uniformly in the [0 1790] MW interval.

As regards the protection, two rules $(k=2)$ have been considered. The measured signal $V$ is the average voltage over five $735-\mathrm{kV}$ buses in the Montréal area. Both the "sum" (2) and the "minmax" (3) objective functions have been considered, for comparison purposes. Requirements 1, 2 and 3 of Section III.C have been taken into account. However, in accordance with Hydro-Québec planning rules, the 2 nd requirement has been amended by allowing some (hopefully small) load shedding to take place after a stable but severe incident. The N-2 scenarios $\mathrm{Nb} .12,17,18$ and 22 are concerned. The latter are merely handled as unstable scenarios with $P_{i}^{\star}=0$ in $(2,3)$.

For coding purposes, the unknown parameters $\mathbf{x}$ were discretized as follows:

- threshold voltages $V^{\text {min }}$ : 8 (equally spaced) values in the range $\left[\begin{array}{ll}0.88 & 0.96\end{array}\right] \mathrm{pu}$. The lower bound is the lowest admissible voltage (the protection is expected to quickly increase $V$ above this value) while the upper bound is just smaller than the MAIS settings (typically 0.965-0.97 pu). The threshold varies by $0.011 \mathrm{pu}$ steps, quite close to the measurement accuracy;

- delays $d_{i}: 16$ values in the range [3 18] s. The lower bound is the minimal delay to distinguish a voltage instability from a temporary undervoltage;

- shedding steps $\Delta P_{3}: 16$ values in the range [50 800] $\mathrm{MW}$. The lower bound is the minimum amount that can be tripped by opening distribution feeders, while the upper bound has been limited to avoid excessive load shedding.

The $\mu \mathrm{GA}$ software of [10] has been used.

\section{Results and discussion}

Minmax objective. The convergence of the $\mu \mathrm{GA}$ is declared after 700 generations, since no significant decrease in the objective has been observed over the last 150 generations. The obtained rules are:

$\mathrm{R} 1$ : if $V<0.95$ pu during 11 seconds, shed $200 \mathrm{MW}$

R2: if $V<0.93$ pu during 4 seconds, shed $500 \mathrm{MW}$

These results can be interpreted by inspection of QSS time simulations of the type shown in Fig. 3, where a star indicates a MAIS operation and $\mathrm{Rx}$ a load shedding due to rule $\mathrm{Rx}$.

Rule $\mathrm{R} 1$ is used in the less severe unstable scenarios, for which $V$ does not decrease below $0.93 \mathrm{pu}$. The rather long delay and the moderate shedding of $\mathrm{R} 1$ yield a good coordination with the MAIS, which are given time to act. For instance, in the case of Fig. 3.a, MAIS operation makes $V$ recover before any of the two rules is triggered. This avoids to shed load in stable scenarios. In the case of Fig. 3.b, load is shed only after all the MAIS have been exhausted.

Rule R2 is used in more severe scenarios, leading to a more pronounced voltage drop. An example is provided in Fig. 3.c. Note that by not shedding too much load, the voltage remains below the settings of the MAIS and the latter operate, adding to the effect of load shedding.

The structure of the above rules is "classical" in the sense that the larger the voltage drop, the greater the action and the smaller the delay to take this action. This is not the case when the sum objective is minimised, as explained hereafter.

Sum objective. The convergence of the $\mu \mathrm{GA}$ is declared after 850 generations. The obtained rules are:

$\mathrm{R} 1:$ if $V<0.93$ pu during 3 seconds, shed $350 \mathrm{MW}$

R2: if $V<0.91$ pu during 4 seconds, shed $250 \mathrm{MW}$

The rather low voltage threshold of rule $\mathrm{R} 1$ guarantees that no load is shed in the stable scenarios (see for instance Fig. 3.d) and allows more load to be shed ( 350 instead of $200 \mathrm{MW}$ ) without preventing the MAIS to operate.

The settings are such that rule $\mathrm{R} 2$ will be used only if the voltage initially drops below $0.91 \mathrm{pu}$ and stays below this value after load has been shed by rule R1. Figure 3.e shows a scenario where the triggering of $\mathrm{R} 1$ inhibits $\mathrm{R} 2$, while in the more severe scenario of Fig. 3.f the two rules are triggered.

In other words, this protection behaves as if it had a single rule but two shedding levels ( 350 and $600 \mathrm{MW}$ resp.), according to the disturbance severity.

Comparison. Figure 4 compares the performances of the two protections in terms of "over-shedding" with respect to the optimal values $P_{i}^{\star}$. The figure relates to the 17 unstable scenarios and the 4 stable $\mathrm{N}-2$ scenarios where load shedding is allowed (in the remaining 15 stable cases, no load has been shed)

As can be seen, the sum objective yields perfect results in some scenarios but also over-sheddings as large as $540 \mathrm{MW}$ in some others. Expectedly, the minmax objective yields more uniform errors, with a maximum over-shedding of $360 \mathrm{MW}$, but it is never perfect.

Finally, in scenarios $12,17,18$ and 22 , the minmax protection sheds load in three cases while the sum protection never does.

\section{CONCLUDING REMARKS}

In this paper the design of automatic load shedding schemes is formulated as a combinatorial optimization problem solved 

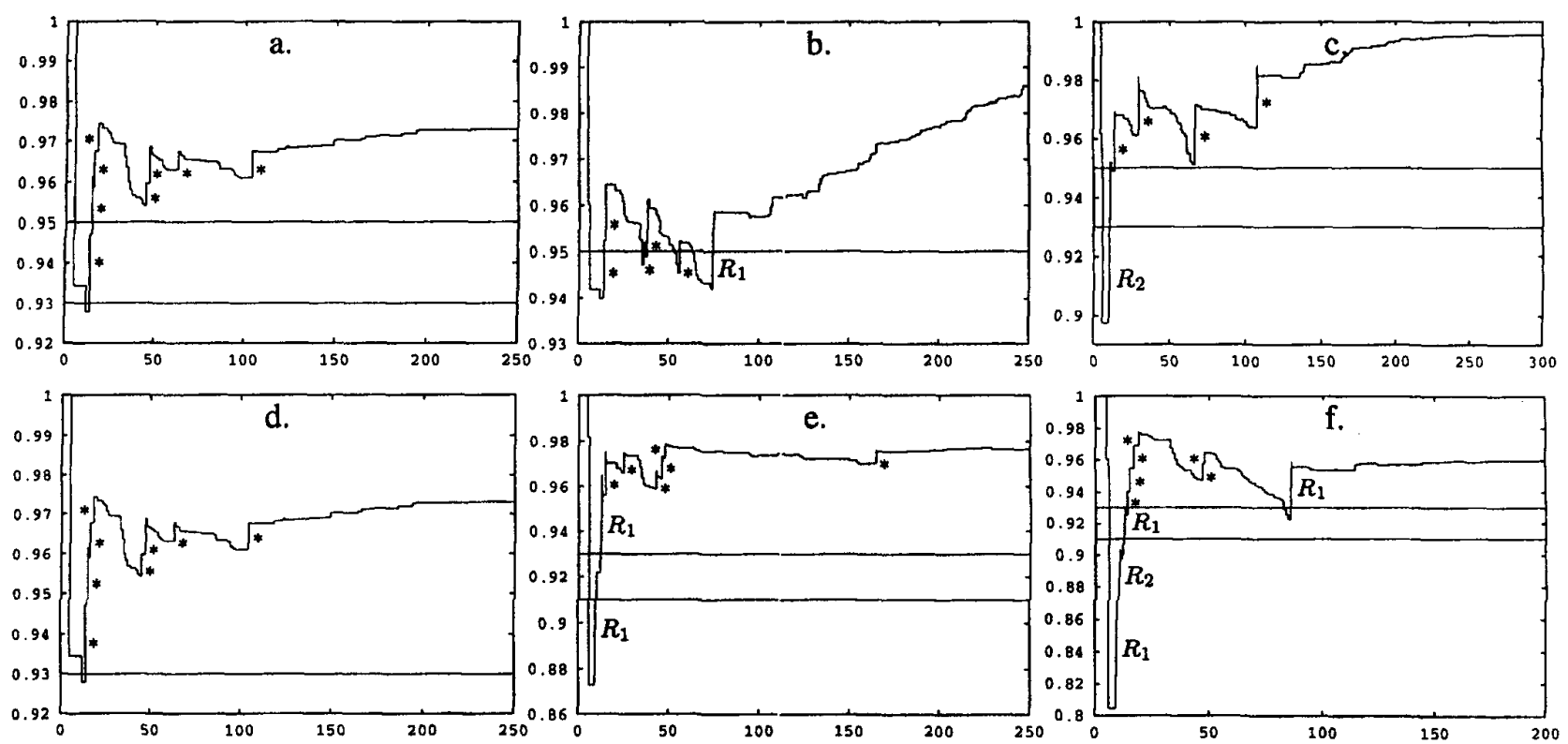

Figure 3: Average voltage in the Montreal area (pu) vs. time (s) in six scenarios stabilized by load shedding and shunt reactor tripping

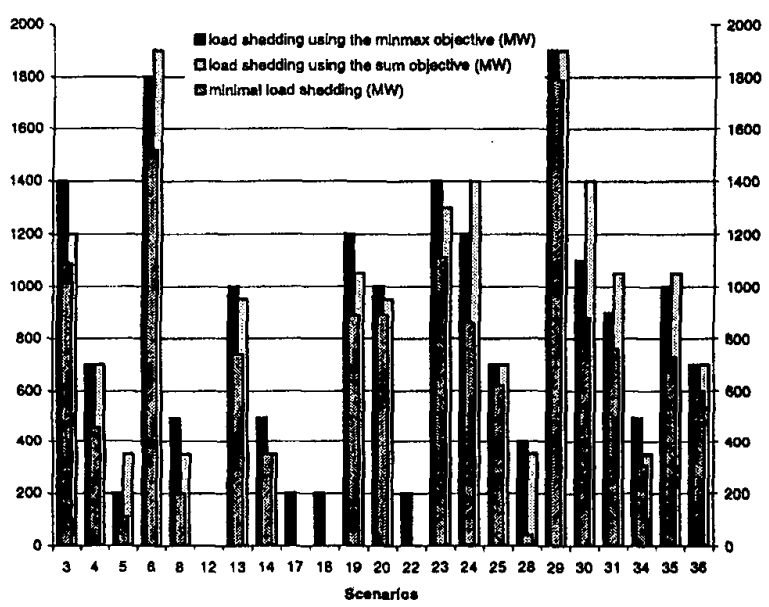

Figure 4: Performances of the sum and minmax objectives

by means of (micro-)genetic algorithms. This yields optimized rules which can be easily implemented and interpreted.

Obviously, many aspects remain to be investigated. Let us quote non exhaustively: a more in-depth tuning of the (micro-)genetic algorithm parameters and the development of techniques to speed up computations. These improvements would allow to handle a larger number of scenarios (e.g. more system configurations and more incidents), a wider range of possible load behaviours (to take into account the uncertainty in their modelling) and eventually more detailed time simulations (in order to handle, for instance, short-term voltage instability situations, or to coordinate load shedding with other, fast countermeasures).
Although Genetic Algorithms have been chosen for their proven ability to deal with complex problems, alternative combinatorial optimization methods are worth being considered.

In spite of the above expected improvements, the approach has already given very satisfactory results. In the Hydro-Québec system, for instance, it is already helping planners in the complex task of designing a robust system protection scheme.

\section{REFERENCES}

[1] C. W. Taylor, Power System Voltage Stability, McGraw Hill, EPRI Power System Engineering series, 1994

[2] T. Van Cutsem, C. Vournas, Voltage Stability of Electric Power Systems, Boston, Kluwer Academic Publishers, 1998

[3] C. W. Taylor, "Concepts of undervoltage load shedding for voltage stability", IEEE Trans. on Power Delivery, Vol. 7, pp. 480-488, 1992

[4] S. Granville, J.C.O. Mello, A.C.G. Melo, "Application of interior point methods to power fiow solvability", IEEE Trans. on Power Systems, Vol. 11, pp. 1096-1104, 1996

[5] C. Moors, T. Van Cutsem, "Determination of Optimal Load Shedding against Voltage Instability", 13th PSCC Proceedings, Trondheim, 1999, pp. 993-1000

[6] T. Van Cutsem, R. Mailhot, "Validation of a fast voltage stability analysis method on the Hydro-Québec system", IEEE Trans. on Power Systems, Vol. 12, pp. 282-292, 1997

[7] G. Trudel, S. Bernard, G. Scott, "Hydro-Québec's defence plan against ex treme contingencies", paper PE-211-PWRS-0-06-1998 presented at the IEEE PES Summer Meeting, San Diego, July 1998

[8] D. E. Goldberg, Genetic Algorithms in search, optimization, and machine learning, Addison-Wesley, Boston, 1989

[9] K. Krishnakumar, "Micro-genetic algorithms for stationary and nonstationary function optimization", Intelligent Control and Adaptative Systems, Proc. SPIE, Vol. 1196, pp. 289-296, 1990

[10] D. L. Carroll, "Fortran Genetic Algorithm Driver", information available from http://www.staff.uiuc.edu/carroll/ga.html

[11] S. Bemard, G. Trudel, G. Scott, "A 735-kV shunt reactors automatic switching system for Hydro-Québec network, IEEE Trans. on Power Systems, Vol. 11, pp. 2024-2030, 1996 